\title{
Probing the top-Yukawa coupling in associated Higgs production with a single top quark
}

\author{
Jung Chang, ${ }^{a}$ Kingman Cheung, ${ }^{a, b}$ Jae Sik Lee ${ }^{c}$ and Chih-Ting Lu ${ }^{a}$ \\ ${ }^{a}$ Department of Physics, National Tsing Hua University, \\ Hsinchu 300, Taiwan \\ ${ }^{b}$ Division of Quantum Phases and Devices, School of Physics, Konkuk University, \\ Seoul 143-701, Republic of Korea \\ ${ }^{c}$ Department of Physics, Chonnam National University, \\ 300 Yongbong-dong, Buk-gu, Gwangju, 500-757, Republic of Korea \\ E-mail: lovejesus99wwjd@gmail.com, cheung@phys.nthu.edu.tw, \\ jslee@jnu.ac.kr, timluyu@yahoo.com.tw
}

ABSTRACT: Associated production of the Higgs boson with a single top quark proceeds through Feynman diagrams, which are either proportional to the $h W W$, top-Yukawa, or the bottom-Yukawa couplings. It was shown in literature that the interference between the top-Yukawa and the gauge-Higgs diagrams can be significant, and thus the measurement of the cross sections can help pin down the sign and the size of the top-Yukawa coupling. Here we perform a detailed study with full detector simulations of such a possibility at the LHC-14 within the current allowed range of $h W W$ and top-Yukawa couplings, using $h \rightarrow b \bar{b}, \gamma \gamma, \tau^{+} \tau^{-}, Z Z^{*} \rightarrow 4 \ell$ modes. We found that the LHC-14 has the potential to distinguish the size and the sign of the top-Yukawa coupling. Among the channels the $h \rightarrow b \bar{b}$ mode provides the best chance to probe the signal, followed by the $h \rightarrow \gamma \gamma$ mode, which has the advantage of a narrow reconstructed mass peak. We also pointed out that the spatial separation among the final-state particles has the potential in differentiating among various values of the top-Yukawa coupling.

Keywords: Higgs Physics, Beyond Standard Model

ARXIV EPRINT: 1403.2053 


\section{Contents}

1 Introduction 1

2 Formalism 2

3 Variation of cross sections 5

$3.1 q b \rightarrow t h q^{\prime}$

$3.2 g q \rightarrow t h q^{\prime} \bar{b} \quad 6$

$3.3 \mathrm{gb} \rightarrow \mathrm{thW} \quad 6$

$3.4 q \bar{q}^{\prime} \rightarrow t h \bar{b} \quad 6$

3.5 Variation of the cross sections versus $C_{t}^{P} \quad 6$

4 Potential at the LHC $\quad 7$

$\begin{array}{llr}4.1 & \text { Semileptonic top decay } & 9\end{array}$

$\begin{array}{lll}4.1 .1 & h \rightarrow b \bar{b} & 10\end{array}$

$\begin{array}{lll}4.1 .2 & h \rightarrow \gamma \gamma & 12\end{array}$

$\begin{array}{lll}\text { 4.1.3 } h \rightarrow \tau^{+} \tau^{-} & 13\end{array}$

$\begin{array}{lll}4.2 & \text { Hadronic top decay } & 16\end{array}$

4.2.1 $h \rightarrow Z Z^{*} \rightarrow 4 \ell \quad 16$

4.3 Distinction among $C_{t}^{S}=1,0,-1 \quad 16$

$\begin{array}{llr}5 & \text { Discussion } & 18\end{array}$

A Amplitude of $q b \rightarrow t h q^{\prime}$ in the effective $W$ approximation 20

\section{Introduction}

It has been established that the Higgs boson has been found at the Large Hadron Collider (LHC) [1, 2]. The measured properties of the Higgs boson are best described by the standard-model (SM) Higgs boson [3], which was proposed in 1960s [4-6]. The study [3] showed that the gauge-Higgs coupling $C_{v} \equiv g_{h W W}=1.01_{-0.14}^{+0.13}$ is very close to the SM value, but the top- and bottom-Yukawa couplings cannot be determined as precise as $C_{v}$ by the current data. In particular, since the Higgs boson cannot decay into a top-quark pair, the top-Yukawa coupling can only be determined as $0.00 \pm 1.18\left(0.80_{-0.13}^{+0.16}\right)$ in the fit that allows (disallows) additional loop contributions to $h \gamma \gamma$ and $h g g$ couplings. ${ }^{1}$ This is easy to understand because the top-Yukawa coupling only appears in the loops of $h \gamma \gamma$

\footnotetext{
${ }^{1}$ If additional loop contributions to $h \gamma \gamma$ and $h g g$ couplings are allowed, the top-Yukawa coupling is only loosely bounded due to a very small contribution of associated Higgs production with a $t \bar{t}$ pair to the current Higgs data.
} 
and $h g g$ in the gluon fusion process, and also because the top contribution is much smaller than the $W$-loop contribution in the $h \gamma \gamma$ coupling. Some other methods to determine the top-Yukawa are desired.

In literature, the most studied process of probing the top-Yukawa is associated Higgs production with a top-quark pair $p p \rightarrow t \bar{t} h$, which can directly determine the absolute value of the top-Yukawa coupling. However, the sign cannot be determined in this process. On the other hand, associated Higgs production with a single top quark has the potential of measuring the sign of the top-Yukawa coupling [7-14]. Just take an example of one of the processes that contribute to a single top quark and a Higgs boson in the final state, $q b \rightarrow t h q^{\prime}$ as shown in figure 1. Diagram (a) is proportional to the gauge-Higgs coupling and the diagram (b) is proportional to the top-Yukawa coupling. There is another diagram with the Higgs boson attached to the bottom-quark leg but is very small proportional to the bottom-Yukawa coupling. The interference between the top-Yukawa diagram and the gauge-Higgs diagram was shown to be significant and induces large variations in the total cross section with the size and the relative sign of the Higgs couplings to the gauge boson and the top quark. Therefore, if in the future the production cross section of a single top quark and a Higgs boson can be measured with sufficient accuracy, one can determine the size and the relative sign of the top-Yukawa coupling. In this work, we study associated Higgs production with a single top quark and the potential of measuring the size and the sign of top-Yukawa in the presence of backgrounds, with full detector simulations. This is the main objective of this work.

In addition to the above process, there are other processes that a single top quark and a Higgs boson can appear in the final state: $q g \rightarrow t h q^{\prime} \bar{b}, g b \rightarrow t h W^{-}$, and $q \bar{q}^{\prime} \rightarrow t h \bar{b}$. Since additional or different particles appear in the final state, all these processes can be specifically identified, although the first process $q b \rightarrow t h q^{\prime}$ has the largest cross section.

In this work, we investigate various processes that contribute to the final states: $t h+X$ with (i) $X=j$, (ii) $X=j+b$, (iii) $X=W$, and (iv) $X=b$. Here top quark $t$ can decay semileptonically or hadronically, and the Higgs boson $h$ can decay into $b \bar{b}, \gamma \gamma, Z Z^{*} \rightarrow 4 \ell$, or $\tau^{+} \tau^{-}$. The $h \rightarrow W W^{*}$ mode is not considered here because of the Higgs boson cannot be fully reconstructed.

The organization is as follows. In the next section, we lay down the formalism and the calculation method. In section 3, we show the variation of cross sections when we vary the couplings. In section 4, we calculate the event rates with detector simulations and estimate the feasibility at the LHC. We discuss and conclude in section 5.

\section{Formalism}

The production processes that contribute to a single top quark and a Higgs boson plus anything else can be found in figures $1-4$. We have marked in particular the vertices of $h W W, h t t$, and $h b b$. The production cross sections depend on the relative size and sign of the gauge-Higgs and Yukawa couplings. Assuming that the Higgs boson is a generic 


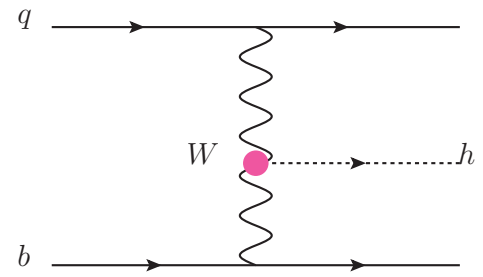

(a)

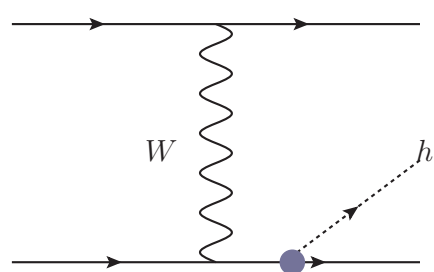

(b)

Figure 1. Contributing Feynman diagrams for $q b \rightarrow t h q^{\prime}$.

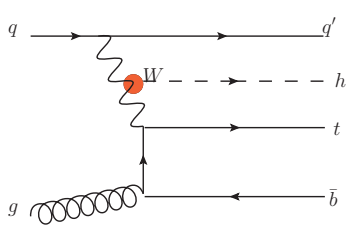

(a)

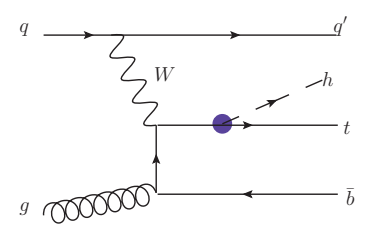

$(b)$

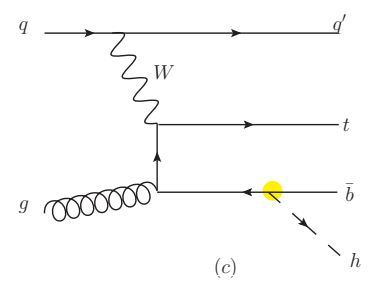

Figure 2. Some of the contributing Feynman diagrams for $q g \rightarrow t h q^{\prime} \bar{b}$.

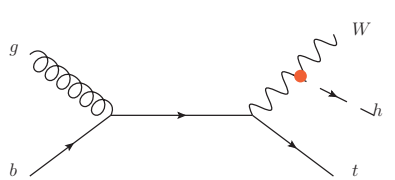

(a)

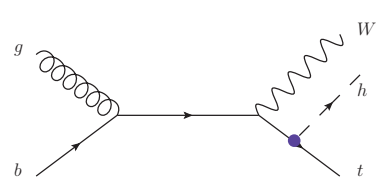

(b)

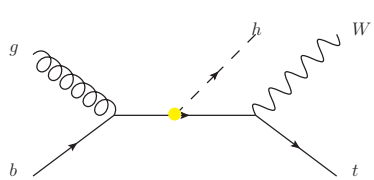

$(c)$

Figure 3. Some of the contributing Feynman diagrams for $g b \rightarrow t h W^{-}$.

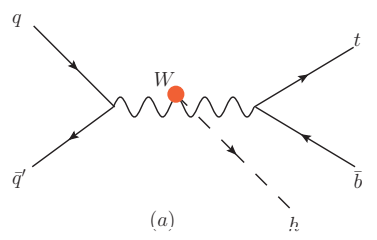

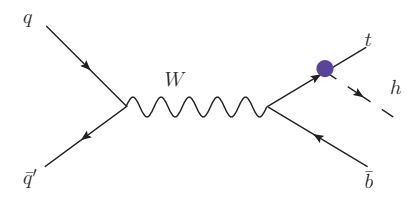

$(b)$

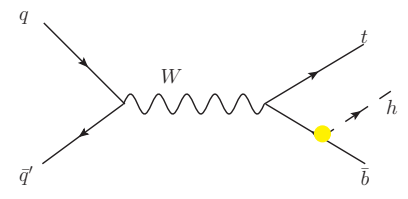

(c)

Figure 4. Contributing Feynman diagrams for $q \bar{q}^{\prime} \rightarrow t h \bar{b}$.

CP-mixed state, we can write the gauge-Higgs and Yukawa couplings as

$$
\begin{aligned}
\mathcal{L}_{h V V} & =g m_{W}\left(g_{h W W} W_{\mu}^{+} W^{-\mu}+g_{h Z Z} \frac{1}{2 c_{W}^{2}} Z_{\mu} Z^{\mu}\right) h, \\
\mathcal{L}_{h f f} & =-\sum_{f=t, b, c, \tau} \frac{g m_{f}}{2 m_{W}} \bar{f}\left(g_{h f f}^{S}+i g_{h f f}^{P} \gamma_{5}\right) f h .
\end{aligned}
$$

Here only $f=t, b$ are relevant to the production cross sections of the processes in figures 14. In the SM, $g_{h W W}=g_{h Z Z}=g_{h f f}^{S}=1$ and $g_{h f f}^{P}=0$. 
In order to calculate the event rates we have to consider the decay branching ratios of the Higgs boson, which depend on $g_{h W W}, g_{h Z Z}, g_{h t t, h b b}^{S, P}$, and a few more couplings, including $h \tau \tau, h c c, h \gamma \gamma$, and $h g g$. The amplitude for the decay process $h \rightarrow \gamma \gamma$ can be written as

$$
\mathcal{M}_{h \gamma \gamma}=-\frac{\alpha m_{h}^{2}}{4 \pi v}\left\{S^{\gamma}\left(m_{h}\right)\left(\epsilon_{1 \perp}^{*} \cdot \epsilon_{2 \perp}^{*}\right)-P^{\gamma}\left(m_{h}\right) \frac{2}{m_{h}^{2}}\left\langle\epsilon_{1}^{*} \epsilon_{2}^{*} k_{1} k_{2}\right\rangle\right\}
$$

where $k_{1,2}$ are the momenta of the two photons and $\epsilon_{1,2}$ the wave vectors of the corresponding photons, $\epsilon_{1 \perp}^{\mu}=\epsilon_{1}^{\mu}-2 k_{1}^{\mu}\left(k_{2} \cdot \epsilon_{1}\right) / m_{h}^{2}, \epsilon_{2 \perp}^{\mu}=\epsilon_{2}^{\mu}-2 k_{2}^{\mu}\left(k_{1} \cdot \epsilon_{2}\right) / m_{h}^{2}$ and $\left\langle\epsilon_{1} \epsilon_{2} k_{1} k_{2}\right\rangle \equiv$ $\epsilon_{\mu \nu \rho \sigma} \epsilon_{1}^{\mu} \epsilon_{2}^{\nu} k_{1}^{\rho} k_{2}^{\sigma}$. Including some additional loop contributions from new particles, the scalar and pseudoscalar form factors, retaining only the dominant loop contributions from the third-generation fermions and $W^{ \pm}$, are given by

$$
\begin{aligned}
& S^{\gamma}\left(m_{h}\right)=2 \sum_{f=b, t, \tau} N_{C} Q_{f}^{2} g_{h f f}^{S} F_{s f}\left(\tau_{f}\right)-g_{h W W} F_{1}\left(\tau_{W}\right)+\Delta S^{\gamma}, \\
& P^{\gamma}\left(m_{h}\right)=2 \sum_{f=b, t, \tau} N_{C} Q_{f}^{2} g_{h f f}^{P} F_{p f}\left(\tau_{f}\right)+\Delta P^{\gamma}
\end{aligned}
$$

where $\tau_{x}=m_{h}^{2} / 4 m_{x}^{2}, N_{C}=3$ for quarks and $N_{C}=1$ for taus, respectively. For the loop functions of $F_{s f, p f, 1}(\tau)$, we refer to, for example, ref. [15]. The additional contributions $\Delta S^{\gamma}$ and $\Delta P^{\gamma}$ are due to additional particles running in the loop. In the SM, $P^{\gamma}=0$ and $g_{h f f}^{S}=g_{h W W}=1$. The amplitude for the decay process $h \rightarrow g g$ can be written as

$$
\mathcal{M}_{H g g}=-\frac{\alpha_{s} m_{h}^{2} \delta^{a b}}{4 \pi v}\left\{S^{g}\left(m_{h}\right)\left(\epsilon_{1 \perp}^{*} \cdot \epsilon_{2 \perp}^{*}\right)-P^{g}\left(m_{h}\right) \frac{2}{m_{h}^{2}}\left\langle\epsilon_{1}^{*} \epsilon_{2}^{*} k_{1} k_{2}\right\rangle\right\}
$$

where $a$ and $b(a, b=1$ to 8$)$ are indices of the eight $\mathrm{SU}(3)$ generators in the adjoint representation. Including some additional loop contributions from new particles, the scalar and pseudoscalar form factors are given by

$$
\begin{aligned}
& S^{g}\left(m_{h}\right)=\sum_{f=b, t} g_{h f f}^{S} F_{s f}\left(\tau_{f}\right)+\Delta S^{g}, \\
& P^{g}\left(m_{h}\right)=\sum_{f=b, t} g_{h f f}^{P} F_{p f}\left(\tau_{f}\right)+\Delta P^{g} .
\end{aligned}
$$

In the SM, $P^{g}=0$ and $g_{h f f}^{S}=1$. In the decays of the Higgs boson, we can see that the partial width into $b \bar{b}$ depends on $g_{h b b}$, that into $W W^{*}$ and $Z Z^{*}$ depends on $g_{h W W, h Z Z}$, and that into $\gamma \gamma$ and $g g$ depends implicitly on all $g_{h W W, h Z Z}, g_{h t t}^{S, P}, g_{h b b}^{S, P}$, and $g_{h \tau \tau}^{S, P}$.

The dependence of the production cross sections and the decay branching ratios on $g_{h W W}$ and $g_{h f f}^{S, P}$ has been explicitly shown in the above equations. Since we are primarily interested in the relative size and sign of the gauge-Higgs and top- and bottom-Yukawa couplings, for bookkeeping purposes we use the following notation

$$
C_{v} \equiv g_{h V V}=g_{h W W}=g_{h Z Z}, \quad C_{t}^{S, P} \equiv g_{h t t}^{S, P}, \quad C_{b}^{S, P} \equiv g_{h b b}^{S, P} .
$$

We will show the variation of the cross sections in the next section. 

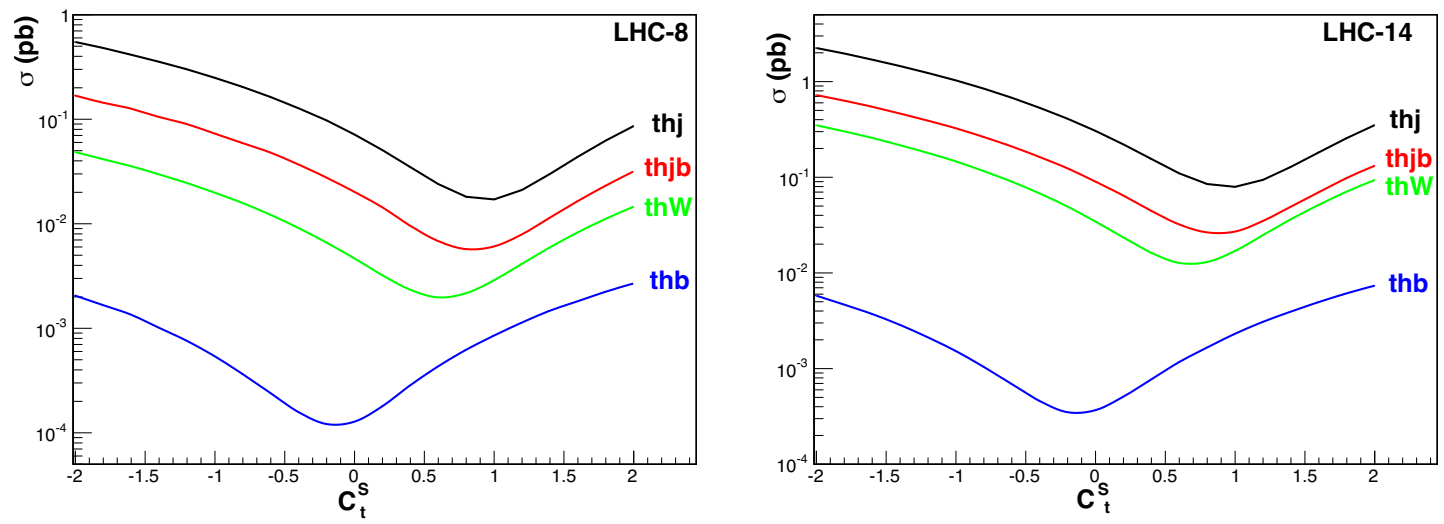

Figure 5. Variation of the total cross sections versus $C_{t}^{S}$ for $p p \rightarrow t h X$ with $X=j, j b, W, b$ in the order of the size of cross sections at (a) LHC-8 and (b) LHC-14. We have taken $C_{v}=C_{b}^{S}=1$ and $C_{t, b}^{P}=0$. No cuts are imposed except for the second process $p p \rightarrow t h j b$ in which we applied the cuts in eq. (3.1) to remove the divergence.

\section{$3 \quad$ Variation of cross sections}

In this section, we show the cross sections of the processes listed in the last section versus the top-Yukawa $C_{t}^{S}$ and $C_{t}^{P}$. We use MADGRAPH [16] with the 5 -flavor scheme $(u, d, s, c, b$ partons) for calculating the cross sections. We do not impose cuts as we are presenting the total cross sections here, except for the process $p p \rightarrow t h j b$, where we have to impose cuts on the final state $b, j$ to remove the divergences. We use CTEQ6 [17] for parton distribution functions with the renormalization/factorization scale equal to $M_{Z}$.

\section{1 $q b \rightarrow t h q^{\prime}$}

The Feynman diagrams are shown in figure 1. We have also included the subprocesses with $\bar{b}$ and all possible $q$ and $\bar{q}$ in the initial state. Therefore, both thj and $\bar{t} h j$ final states are included. It is clear that both couplings $C_{v}$ and $C_{t}^{S, P}$ can affect the total cross sections, while $C_{b}^{S}$ has a negligible effect because of the small $b$-quark mass.

In figure 5 we show the total cross sections of $p p \rightarrow t h j$ (the curve at the top) versus $C_{t}^{S}$ at the LHC-8 and LHC-14, and we have fixed $C_{v}=1$ and $C_{t}^{P}=0$. It is clear that the cross section depends crucially on the value of $C_{t}^{S}$. The minimum cross section of $t h j$ appears very near the SM value of $C_{t}^{S}=1$. The cross section keeps increasing for $C_{t}^{S}$ decreasing from 1 and for $C_{t}^{S}$ increasing from 1 . On the other hand, the effect of $C_{b}^{S, P}$ on the production cross section is very small. ${ }^{2}$ In addition to the figure, we also show the cross sections for these four processes in table 1 for $C_{t}^{S}=1,0,-1$. It is clear that the size of the cross sections decreases as $X=j>X=j b>X=W>X=b$.

\footnotetext{
${ }^{2}$ The cross section multiplied the branching ratio $\sigma(p p \rightarrow t h j) \times B(h \rightarrow b \bar{b})$ strongly depends on $C_{b}^{S, P}$, because the partial width into $b \bar{b}$ is directly proportional to $\beta^{2}\left|C_{b}^{S}\right|^{2}+\left|C_{b}^{P}\right|^{2}$ with $\beta^{2}=1-4 m_{b}^{2} / m_{h}^{2}$. Other processes have similar features with the variation in $C_{b}^{S, P}$. The production cross section itself shows very small effects from $C_{b}^{S, P}$, but the cross section multiplied by the branching ratio $\sigma \times B(h \rightarrow b \bar{b})$ varies significantly with $C_{b}^{S, P}$.
} 


\begin{tabular}{|lcccc|}
\hline & \multicolumn{4}{c|}{$\sigma(p p \rightarrow t h X)[\mathrm{fb}]$} \\
& $X=j$ & $X=j+b$ & $X=W$ & $X=b$ \\
\hline$C_{t}^{S}=+1(\mathrm{SM})$ & $79.4(17.1)$ & $27.1(5.95)$ & $17.0(2.89)$ & $2.32(0.833)$ \\
$C_{t}^{S}=0$ & $305(71.4)$ & $90.0(19.8)$ & $34.4(4.66)$ & $0.368(0.126)$ \\
$C_{t}^{S}=-1$ & $1030(249)$ & $325(72.8)$ & $146(19.8)$ & $1.52(0.536)$ \\
\hline
\end{tabular}

Table 1. The leading-order production cross sections in $\mathrm{fb}$ for the processes $p p \rightarrow t h+X$ at $14 \mathrm{TeV}(8 \mathrm{TeV}) \mathrm{LHC}$, taking $C_{v}=C_{b}^{S}=1$ and $C_{t, b}^{P}=0$. We have not applied any cuts except for the case with $X=j+b$ for which we required $p_{T_{b}}>25 \mathrm{GeV},\left|\eta_{b}\right|<2.5 ; p_{T_{j}}>10 \mathrm{GeV},\left|\eta_{j}\right|<5$, see text for details.

\section{2 $g q \rightarrow t h q^{\prime} \bar{b}$}

Some of the contributing Feynman diagrams are shown in figure 2. Both thj $\bar{b}$ and $\bar{t} h j b$ final states are included. Note that one can regard this process as a higher-order correction to the process $q b \rightarrow t h q^{\prime}$ in the previous subsection when we do not tag the $b$-quark in the final state. In order to distinguish them, we impose a minimal set of cuts on the $b$ and $j$ (also needed to avoid the collinear divergence):

$$
p_{T_{b}}>25 \mathrm{GeV}, \quad\left|\eta_{b}\right|<2.5 ; \quad p_{T_{j}}>10 \mathrm{GeV}, \quad\left|\eta_{j}\right|<5 .
$$

Also, this set of cuts is used to avoid the double-counting of the cross section against the process $q b \rightarrow t h q^{\prime}$ in the previous subsection. We show the variation of the cross sections of $p p \rightarrow t h j b$ (the second curve from the top) versus $C_{t}^{S}$ at the LHC-8 and LHC-14 in figure 5. The minimum cross section occurs at about $C_{t}^{S}=+0.85$, and the cross section increases approximately symmetric about this minimum point.

\section{3 $g b \rightarrow t h W$}

Some of the contributing Feynman diagrams are shown in figure 3. Both $t h W^{-}$and $\bar{t} h W^{+}$ final states are included. We show the variation of the cross sections of $p p \rightarrow t h W$ (the third curve from the top) versus $C_{t}^{S}$ at the LHC-8 and LHC-14 in figure 5. The minimum cross section occurs at about $C_{t}^{S}=+0.6$, and the cross section increases approximately symmetric about this minimum point.

\section{$3.4 \quad q \bar{q}^{\prime} \rightarrow t h \bar{b}$}

The Feynman diagrams are shown in figure 4. Both $t h \bar{b}$ and $\bar{t} h b$ final states are included. We show the variation of the cross sections of $p p \rightarrow t h b$ (the bottom curve) versus $C_{t}^{S}$ at the LHC-8 and LHC-14 in figure 5. The minimum cross section occurs at about $C_{t}^{S}=-0.15$, which is far from the SM value, and the cross section increases approximately symmetric about this minimum point. Being different from the three processes considered before, $q \bar{q}^{\prime} \rightarrow t h b$ is an $s$-channel process mediated by a mostly off-shell $W$.

\subsection{Variation of the cross sections versus $C_{t}^{P}$}

So far we only concern the scalar component in the top-Yukawa coupling. In eq. (2.2), we can also have the pseudoscalar component in the coupling, which is proportional to 
$i \gamma^{5} g_{h f f}^{P}$. In this subsection, we examine the variation of the cross sections when the pseudoscalar component is present in the $h t \bar{t}$ vertex. It was shown in ref. [3] that the scalar and pseudoscalar components in $h t \bar{t}$ are constrained nontrivially, as shown in figure 10(c) of ref. [3]. The $C_{t}^{S}$ and $C_{t}^{P}$ are roughly constrained by an elliptical equation, given by [3]

$$
1=\frac{\left(C_{t}^{S}\right)^{2}}{(0.86)^{2}}+\frac{\left(C_{t}^{P}\right)^{2}}{(0.56)^{2}} .
$$

We can parameterize $C_{t}^{S}$ and $C_{t}^{P}$ by

$$
C_{t}^{S}=0.86 \cos \theta ; \quad C_{t}^{P}=0.56 \sin \theta .
$$

The actual angle $\phi$ presented in the plane of $\left(C_{t}^{S}, C_{t}^{P}\right)$ is related to $\theta$ by

$$
\tan \phi \equiv \frac{C_{t}^{P}}{C_{t}^{S}}=\frac{0.56 \sin \theta}{0.86 \cos \theta}=0.66 \tan \theta
$$

where the ranges of $\phi$ is $-\pi \leq \phi<\pi$. Nevertheless, if we restrict to the $68 \%$ C.L. region of the figure10(c) of ref. [3], the range of allowed $\phi$ is approximately $-2 \pi / 3 \leq \phi \leq 2 \pi / 3$. We show the cross sections versus $\phi$ in figure 6 , in which the shaded regions are those disallowed at $68 \%$ C.L. obtained in ref. [3]. It is interesting to note that the first three curves at the top of the figure have similar behavior across $\phi$ while the bottom curve has the opposite behavior. Again it is due to the $s$-channel exchange mediated by a mostly off-shell $W$ in the last process.

One comment about the next-to-leading order (NLO) corrections is in order here. Since the NLO QCD corrections to single-top plus Higgs production are very similar to singletop production, we can roughly estimate the QCD corrections to the current processes by looking up the NLO corrections to single-top production. A number of NLO and next-nextto-leading order calculations existed in literature for single top-quark production [18-21]. The NLO corrections to the process $q b \rightarrow t q^{\prime}$ and $q g \rightarrow t q^{\prime} \bar{b}$ are very modest, usually less than $10 \%$, while those of $g b \rightarrow t W^{-}$and $q \bar{q}^{\prime} \rightarrow t \bar{b}$ can be as large as $40-50 \%$. We shall estimate the potential at the LHC using the process $q b \rightarrow t h q^{\prime}$, which has the largest cross section among the signal processes, and therefore the NLO correction on the signal cross section is a mere less than $10 \%$ effect.

\section{Potential at the LHC}

We have demonstrated in the previous section that when we change $C_{t}^{S}$ the production cross sections change significantly. If one can measure the event rates of associated Higgs production with a single top quark, the size and sign of $C_{t}^{S}$ can be determined. There are 4 production processes of a Higgs boson and a single top at the LHC: $p p \rightarrow$ thX with $X=j, X=j+b, X=W$, and $X=b$. The top quark and the Higgs boson decay subsequently. Including the semileptonic and hadronic decays of top quark and the five Higgs decay modes into $b \bar{b}, \tau^{+} \tau^{-}, W W^{*}, Z Z^{*}$, and $\gamma \gamma$, we have 40 channels which require different search strategies against different backgrounds. 

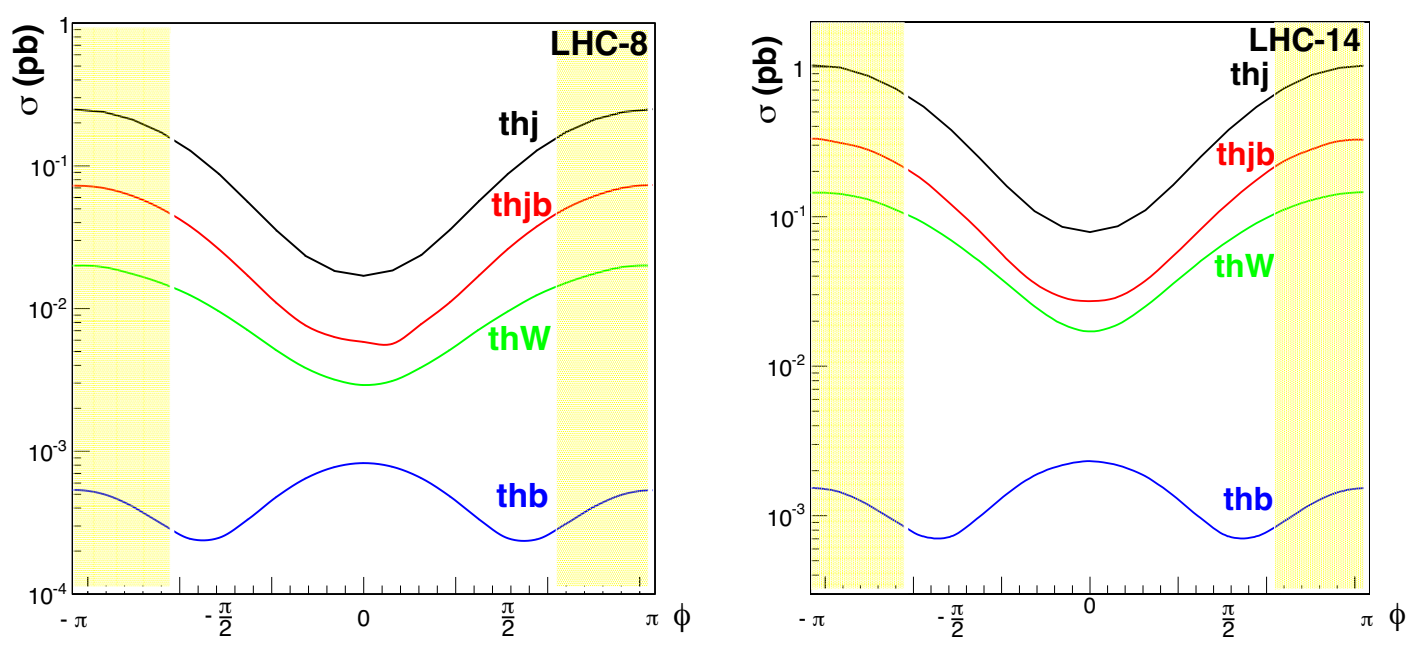

Figure 6. Production cross sections at the LHC-14 for $p p \rightarrow t h j$ versus $\phi=\arctan \left(C_{t}^{P} / C_{t}^{S}\right)$ under the constraint $\left(C_{t}^{S} / 0.86\right)^{2}+\left(C_{t}^{P} / 0.56\right)^{2}=1$. We take $C_{v}=1$. The shaded regions are those disallowed at $68 \%$ C.L. by the Higgs data obtained in ref. [3].

In this section, we look at a few decay channels of the Higgs boson and investigate the feasibility of isolating the signal events in the presence of backgrounds after implementing detector simulations. A few decay channels that we shall study are: $h \rightarrow b \bar{b}, h \rightarrow \gamma \gamma$, $h \rightarrow \tau^{+} \tau^{-}$, and $h \rightarrow Z Z^{*} \rightarrow 4 \ell$. These are the channels enable one to reconstruct the Higgs boson, especially the $h \rightarrow \gamma \gamma$ and $h \rightarrow Z Z^{*} \rightarrow 4 \ell$, which can help reducing the backgrounds by imposing the invariant-mass cut on $M_{\gamma \gamma}$ or $M_{4 \ell}$. The other two channels $h \rightarrow \tau \tau, b \bar{b}$ are not as effective as $\gamma \gamma$ and $Z Z^{*} \rightarrow 4 \ell$ channels in reconstructing the invariant mass. We delay the channel $h \rightarrow W W^{*} \rightarrow \ell \nu \ell \nu$ to later studies. For the top quark decay we can choose either the semileptonic or hadronic decay, depending on how complicated the final state will be. For example, if $h \rightarrow b \bar{b}$ we only choose the semileptonic decay for the top quark. If we choose a non-hadronic decay mode for the Higgs boson, we can afford the luxury to have both the semileptonic and hadronic decays of the top quark.

We calculate the signal and background processes and generate events by MADGRAPH [16], perform parton showering by Pythia [22], and employ the detector simulations by Delphes 3 [23]. We will give details about the selection cuts, detection efficiencies, and signal and background event rates in the next few subsections. For easy reading we summarize the detection efficiencies for $b$ quarks [24], $\tau$ leptons [25, 26], charged leptons ( $\mu$ and $e$ ), and photons in table 2 , as well as the mis-tag probabilities for the charm quark to fake a $b$-jet, other light quarks or gluon to fake a $b$-jet [24], mis-tag probability for a jet to fake a tau lepton, and that of a jet to fake a photon [27]. ${ }^{3}$ For simplicity we assume the efficiencies are constant over a large range of transverse momentum $p_{T}$ larger than

\footnotetext{
${ }^{3}$ The $B$ tagging efficiency can be as high as $85 \%$ but at that point the corresponding mis-tag probability goes up quickly [24]. We take moderate values for both quantities in this study. If we take a smaller $\epsilon_{b}=0.6$, the mis-tag probabilities goes down as $P_{u d s g \rightarrow b}=0.004$ and $P_{c \rightarrow b}=0.08$.
} 


\begin{tabular}{|cccc|}
\hline \multicolumn{4}{|c|}{ Detection efficiencies } \\
\hline$\epsilon_{b}$ & $\epsilon_{\tau}$ & $\epsilon_{\ell}$ & $\epsilon_{\gamma}$ \\
$0.7(0.6)$ & 0.5 & 1.0 & 1.0 \\
\hline \multicolumn{4}{|c|}{ Mistag probability } \\
\hline$P_{c \rightarrow b}$ & $P_{u d s g \rightarrow b}$ & $P_{j \rightarrow \tau}$ & $P_{j \rightarrow \gamma}$ \\
$0.2(0.08)$ & $0.015(0.004)$ & 0.01 & $10^{-3}$ \\
\hline
\end{tabular}

Table 2. The detection efficiencies taken in this work for $b$ quarks, $\tau$ leptons, charged leptons $(\mu$ and $e$ ), and photons, as well as the mis-tag probabilities for other light quarks to fake a $b$-jet or a $\tau$ lepton [24-26]. We also list the probability for a jet faking a photon [27]. The $B$ Tagging efficiency and mis-tag probabilities are correlated. The numbers in parenthesis are for $\epsilon_{b}=0.6$.

the acceptance cut (e.g. $p_{T}>25 \mathrm{GeV}$ ). The efficiencies for charged leptons ( $e$ and $\mu$ ) and photons are more than $90 \%$, and so we simply assume them to be 1 .

We mainly focus on the production with the subprocess $q b \rightarrow t h q^{\prime}$ (figure 1) because its cross section is the largest. The processes in figures 3 and 4 are much smaller at the LHC-14. The process in figure 2 is similar to the first process, and has a cross section about $20-30 \%$ of that of the first process and also one additional $b$ quark in the final state. Specifically, we consider the processes: $p p \longrightarrow t(\rightarrow b l \nu)+h\left(\rightarrow b b, \gamma \gamma, \tau^{+} \tau^{-}\right)+j$ and $p p \longrightarrow t\left(\rightarrow b j_{1} j_{2}\right)+h\left(\rightarrow Z Z^{*} \rightarrow 4 \ell\right)+j$. We find that the hadronically decaying top channel with $h \rightarrow \gamma \gamma$ is less efficient than the semileptonic one and we present only the latter case.

\subsection{Semileptonic top decay}

In this subsection, we consider top-Higgs associated production thj with the single top decaying semileptonically

$$
p p \longrightarrow t h j \rightarrow(b l \nu) h j .
$$

At this stage, we apply a set of basic cuts

$$
\begin{array}{rlrl}
\Delta R_{i j} & >0.4 & & \text { with } i, j \text { denoting } b, j, \text { and } \ell \\
p_{T_{b}} & >25 \mathrm{GeV}, & \left|\eta_{b}\right|<2.5 \\
p_{T_{\ell}} & >25 \mathrm{GeV}, & \left|\eta_{\ell}\right|<2.5 \\
p_{T_{j}} & >25 \mathrm{GeV}, & \left|\eta_{j}\right|<4.7
\end{array}
$$

The spatial separation among the objects (the $b$ jets, the jet, and the lepton) in the final state is denoted by $\Delta R_{i j}$.

If we look at the Feynman diagrams of the subprocess $q b \rightarrow t h q^{\prime}$ (figure 1), the dominant contribution comes from where the intermediate $W$ is almost on-shell, which implies that the incoming $q$ behaves like a spectator and therefore it tends to go forward. This behavior is similar to those encountered in $W W$ scattering [28]. In figure 7(a), we show the spectra of the pseudorapidity of the forward jet for $C_{t}^{S}=1,0,-1$. All the three curves indeed show the forward behavior. We therefore impose the forward-jet requirement. Another useful cut is on the invariant mass of the $b$ quark and the charged lepton $\ell$ coming from the top quark decay. Thus, the invariant mass should always be less than $m_{t}$. We 

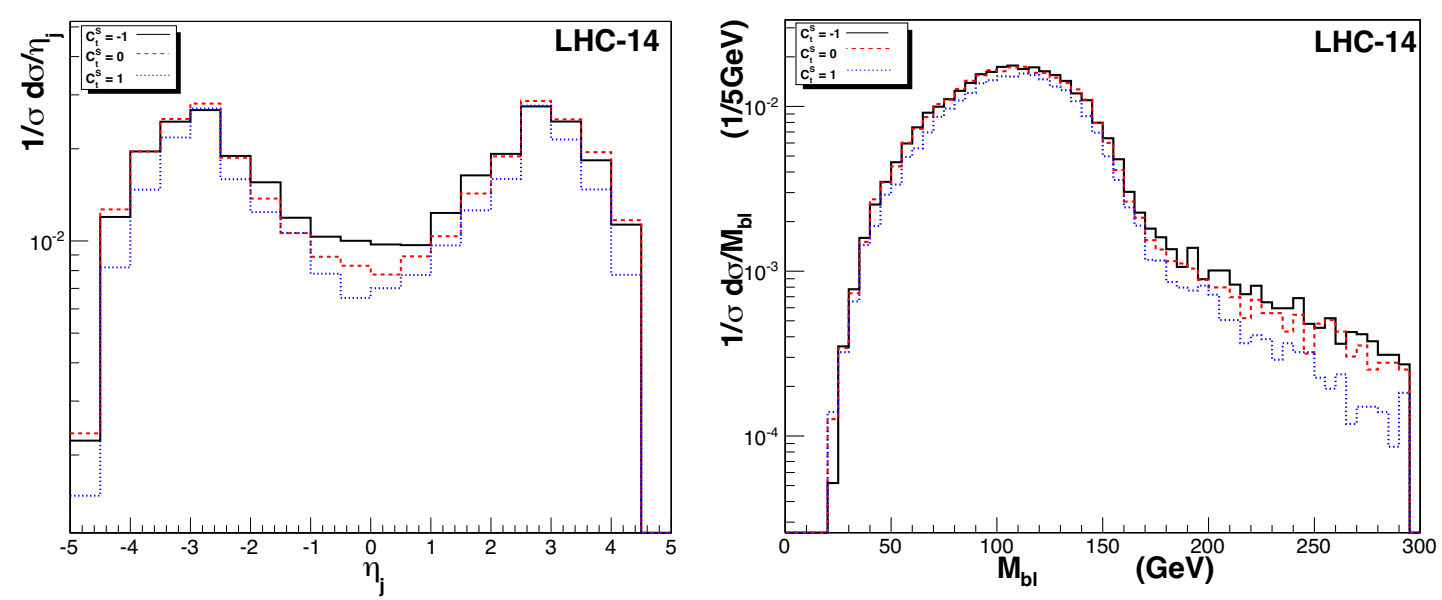

Figure 7. (a) Normalized spectra for the $\eta_{j}$ distribution and (b) normalized invariant mass spectra $M_{b l}$ of the process $p p \rightarrow t h j$ at the LHC-14. We have imposed the set of basic cuts in eq. (4.2) with detector simulations.

show in figure $7(\mathrm{~b})$ the invariant mass spectra $M_{b l}$ for $C_{t}^{S}=1,0,-1$ with detector simulation. After the set of basic cuts listed in eq. (4.2), we further require the forward jet-tag and invariant mass cut on $M_{b l}$, given by

$$
\begin{aligned}
2.5 & <\left|\eta_{j}\right|<4.7, \\
M_{b l} & <200 \mathrm{GeV} .
\end{aligned}
$$

The next level of cuts will depend on the decay channel of the Higgs boson.

\subsection{1 $h \rightarrow b \bar{b}$}

We start with the most difficult decay mode of the Higgs boson because of the large hadronic background. Therefore, with the first process in mind the final state consists of $3 b$ quarks, one charged lepton, and a missing energy due to the neutrino. ${ }^{4}$ The charged lepton with the corresponding $b$ quark from the top decay can be selected with high purity by choosing the smallest $M_{b \ell}$ among the three combinations. The other two $b$ quarks are then considered the $b$ quarks coming from the Higgs boson decay, and can be reconstructed at the Higgs boson mass. For all three $b$ quarks including those from decaying Higgs, we impose the same cuts on their momenta and rapidities as in eq. (4.2).

The major reducible and irreducible backgrounds are QCD production of

(i) $t \bar{t} \rightarrow t\left(\bar{b} j_{1} j_{2}\right) \rightarrow t b \bar{b} j \quad$ with mis-tagging one of $j_{1}$ and $j_{2}$ as $b$,

(ii) $t \bar{t} j \rightarrow t\left(\bar{b} j_{1} j_{2}\right) j \rightarrow t b \bar{b} j$ with mis-tagging one of $j_{1}$ and $j_{2}$ as $b$ and missing the other one,

(iii) $t b \bar{b} j$, and $(i v) t Z j \rightarrow t b \bar{b} j$

\footnotetext{
${ }^{4}$ In ref. [11], the process $p p \rightarrow t h j b$ was studied with the final state containing $4 b$ jets. They showed that the sensitivity is better than that of the process $p p \rightarrow t h j$ with $3 b$ jets in the final state. However, a full detector simulation is needed to establish the statement.
} 


\begin{tabular}{|lccccc|}
\hline Cuts & \multicolumn{3}{c}{ Signals (fb) } & \multicolumn{3}{c|}{ Backgrounds (fb) } \\
& $C_{t}^{S}=1$ & $C_{t}^{S}=0$ & $C_{t}^{S}=-1$ & $t \bar{t}$ & $t \bar{t} j$ \\
\hline (1) Basic cuts eq. (4.2) and & & & & & \\
$p_{T_{b_{1,2}}}>25 \mathrm{GeV},\left|\eta_{b_{1,2}}\right|<2.5$ & 0.793 & 4.23 & 15.29 & 655 & 797 \\
$(2) 2.5<\left|\eta_{j}\right|<4.7$ & 0.388 & 2.20 & 7.68 & 46.2 & 95.6 \\
$(3)\left(M_{b l}\right)^{\text {min }}<200 \mathrm{GeV}$ & 0.387 & 2.19 & 7.59 & 46.2 & 95.6 \\
$(4)\left|M_{b_{1} b_{2}}-m_{h}\right|<15 \mathrm{GeV}$ & 0.13 & 0.74 & 2.5 & 6.69 & 15.2 \\
$(5) M_{b_{1} b_{2} j}>300 \mathrm{GeV}$ & 0.06 & 0.3 & 0.9 & 1.34 & 5.41 \\
\hline$S / \sqrt{S+B}$ for $300 \mathrm{fb}^{-1}$ & 0.40 & 2.0 & 5.6 & & \\
\hline
\end{tabular}

Table 3. The cut flow of cross sections in fb at the LHC-14 for the signal $p p \rightarrow t h j$ with semileptonic decay of the top quark and $h \rightarrow b \bar{b}$, and various backgrounds. We have used the $B$-tag efficiency $\epsilon_{b}=0.6$, mis-tag $P_{c \rightarrow b}=0.08$ and $P_{u d s g \rightarrow b}=0.004$.

In addition to the basic cuts as in eqs. (4.2) and (4.3), we further impose the following selection cuts:

$$
\begin{aligned}
\left|M_{b_{1} \bar{b}_{2}}-m_{h}\right| & <15 \mathrm{GeV}, \\
M_{b_{1} \bar{b}_{2} j} & >300 \mathrm{GeV},
\end{aligned}
$$

to separate the signal events from backgrounds. Here, $b_{1,2}$ denote the bottom quarks which are supposedly coming from the Higgs boson in the signal process while we identify the bottom quark $b$ from the decaying top with the smallest $M_{b l}$, as we have mentioned above, on which we then put the cut $M_{b l}<200 \mathrm{GeV}$.

We require the correct $b_{1} \bar{b}_{2}$ pair to satisfy the Higgs mass window of $\pm 15 \mathrm{GeV}$. We note that we cannot take a smaller window because of the wide spreading of the Higgs peak with detector simulation, in contrast to parton-level studies. We will show the invariant mass spectrum of the $b \bar{b}$ pair shortly. The forward jet-tag is used because of the forward nature of the accompanying jet in the signal process. Finally, we used a cut on the invariant mass $M_{b_{1} \bar{b}_{2} j}>300 \mathrm{GeV}$ of the $b \bar{b}$ pair coming from the Higgs decay and the accompanying jet.

We show the cut flow of cross sections for the signals and backgrounds at the LHC-14 in table 3 . The cross sections shown are calculated with the $B$-tagging efficiency $\epsilon_{b}=0.6$ and mis-tag probabilities $P_{c \rightarrow b}=0.08$ and $P_{u d s g \rightarrow b}=0.004$. We found that the set of probabilities with $\epsilon_{b}=0.7$ would give a somewhat smaller significance, because of much larger mis-tag probabilities. We only include the two most significant backgrounds in this study, namely, the $t \bar{t}$ and $t \bar{t} j$ backgrounds. The other few backgrounds ( $t b \bar{b} j, t Z j$, etc) are substantially smaller than these two and so would not affect the estimates of significance here. The $t \bar{t} j$ background turns out to be the largest background in this channel, because the addition jet in the matrix-element level can be highly energetic, in contrast to the jet activities coming from showering.

One of the most crucial cuts is the invariant mass cut on the $b \bar{b}$ pair coming from the Higgs boson decay. In parton-level, this cut would be $100 \%$ efficient for the signal and can cut away a very large fraction of the backgrounds. However, with detector reconstruction 


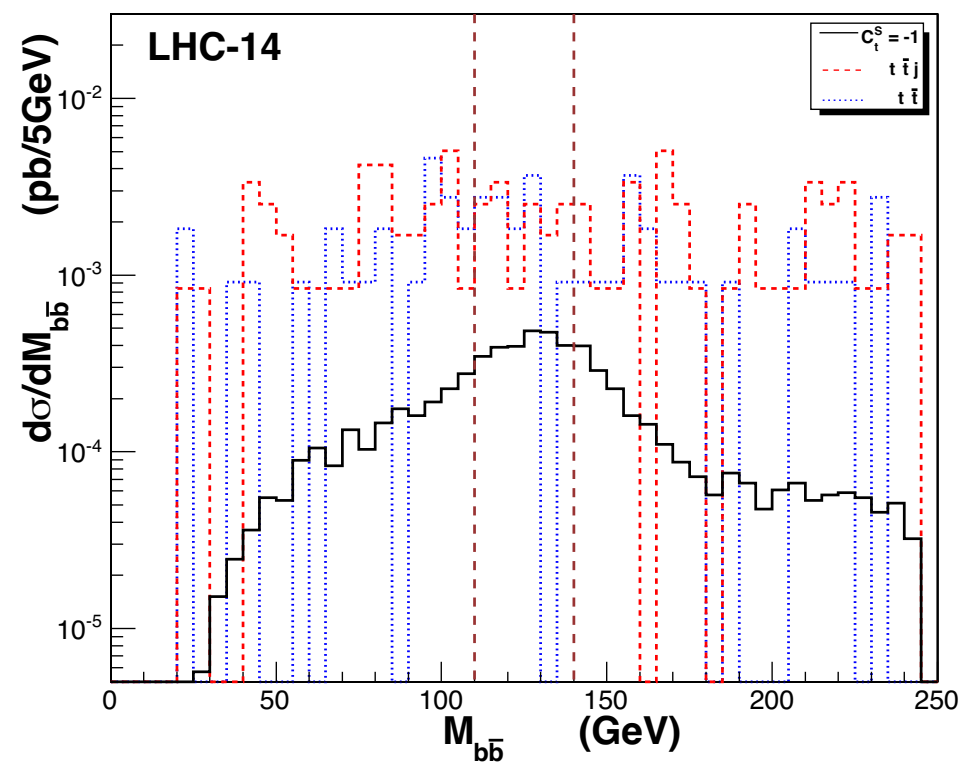

Figure 8. Invariant mass $M_{b \bar{b}}$ distributions for the signal $p p \rightarrow t h j$ with $C_{t}^{S}=-1$ followed by the semileptonic decay of the top quark and $h \rightarrow b \bar{b}$, and for the $t \bar{t}$ and $t \bar{t} j$ backgrounds at the LHC-14. The shape of the peak for $C_{t}^{S}=1,0$ is the same as the one shown with $C_{t}^{S}=-1$. The selection cuts up to level (3) of table 3 have been applied. The two vertical dashed lines are $m_{h} \pm 15 \mathrm{GeV}$.

the invariant mass peak is largely spread out so that we cannot employ a very narrow cut. The invariant mass distributions are shown for the signals and backgrounds in figure 8. Another interesting cut is the forward jet cut, as have been explained above. Finally, the cut on $M_{b \bar{b} j}$ with the $b \bar{b}$ pair from Higgs-boson decay is also effective in reducing the backgrounds.

In table 3, the significance of the signal is also shown for an integrated luminosity of $300 \mathrm{fb}^{-1}$. The $S / \sqrt{S+B}$ ratio can be as high as 5.6 for $C_{t}^{S}=-1$, but however, it decreases rapidly to only 0.4 for $C_{t}^{S}=1$. The signal event rates are about $18-270$ for $C_{t}^{S}=1$ to -1 with an integrate luminosity of $300 \mathrm{fb}^{-1}$.

\subsection{2 $h \rightarrow \gamma \gamma$}

Diphoton decay mode of the Higgs boson is one of the two cleanest channels of the Higgs boson, which allows a sharp reconstructed peak right at the Higgs boson mass, and also makes the background easier to handle. The disadvantage is that the branching ratio is small, of order $10^{-3}$, in the SM. In this study, we employ a fixed branching ratio for $h \rightarrow \gamma \gamma$ at the SM value: $B(h \rightarrow \gamma \gamma)=2.3 \times 10^{-3}$, because there could be extra particles running in the loop that affect the branching ratio. We take a conservative approach for the branching ratio. ${ }^{5}$ Furthermore, we found that it is still easier to handle the backgrounds with the semileptonic decay of the top quark.

\footnotetext{
${ }^{5}$ Changing the value of $C_{t}^{S}$ can also affect the decay branching ratio of $h \rightarrow \gamma \gamma$, because the decay proceeds via a triangular loop of the $W$ boson and the fermions dominated by the top quark. In the SM, the contributions from the $W$ boson and the top quark partially cancel each other. Therefore, when the sign of the top-Yukawa is reversed, these two contributions enhance each other. The branching ratios for $h \rightarrow \gamma \gamma$ with $C_{t}^{S}=1,0,-1$ are $B(h \rightarrow \gamma \gamma)=(2.3,3.7,5.4) \times 10^{-3}$, where we have normalized the SM value of $B(h \rightarrow \gamma \gamma)$ to the value given in ref. [29].
} 


\begin{tabular}{|c|c|c|c|c|c|c|c|}
\hline & \multicolumn{3}{|c|}{ Signals $\left(10^{-3} \mathrm{fb}\right)$} & \multicolumn{4}{|c|}{ Backgrounds $\left(10^{-3} \mathrm{fb}\right)$} \\
\hline & $C_{t}^{S}=1$ & $C_{t}^{S}=0$ & $C_{t}^{S}=-1$ & $t j \gamma \gamma$ & $t j j \gamma$ & $W b j \gamma \gamma$ & $W j j \gamma \gamma$ \\
\hline \multicolumn{8}{|l|}{ (1) Basic cuts eq. (4.2) } \\
\hline and $p_{T_{\gamma}}>20 \mathrm{GeV},\left|\eta_{\gamma}\right|<2.5$ & 4.45 & 22.7 & 80.0 & 318 & 2.59 & 10.5 & 217 \\
\hline (2) $2.5<\left|\eta_{j}\right|<4.7$ & 2.35 & 13.1 & 45.2 & 164 & 0.650 & 1.04 & 20.5 \\
\hline (3) $M_{b l}<200 \mathrm{GeV}$ & 2.30 & 12.7 & 43.6 & 162 & 0.609 & 0.609 & 11.2 \\
\hline (4) $\left|M_{\gamma \gamma}-m_{h}\right|<5 \mathrm{GeV}$ & 1.83 & 10.2 & 34.7 & 5.77 & 0.027 & 0.018 & 0.661 \\
\hline$S / \sqrt{S+B}$ for $300 \mathrm{fb}^{-1}$ & 0.35 & 1.4 & 3.0 & & & & \\
\hline
\end{tabular}

Table 4. The cut flow of cross sections in $10^{-3} \mathrm{fb}$ at the LHC-14 for the signal $p p \rightarrow t h j$ with semileptonic decay of the top quark and $h \rightarrow \gamma \gamma$, and various backgrounds. We have used a $B$-tag efficiency $\epsilon_{b}=0.6$, mis-tag $P_{c \rightarrow b}=0.08$ and $P_{u d s g \rightarrow b}=0.004$, and the jet-fake rate $P_{j \rightarrow \gamma}=10^{-3}$. We employ a fixed branching ratio for $B(h \rightarrow \gamma \gamma)=2.3 \times 10^{-3}$.

The $\gamma \gamma$ decay channel has the great advantage that most QCD backgrounds are gone. The most relevant background comes from $t j \gamma \gamma$ where the photon pair is produced in the continuum. Inside detectors a hadronic jet sometimes can fake a photon with a probability $O\left(10^{-3}\right)$. Therefore, $t j j \gamma$ is a background when one of the jets fakes a photon. Other backgrounds include $W b j \gamma \gamma$ and $W j j \gamma \gamma$. They are all listed in table 4 . Since the spreading of the invariant-mass peak at $m_{h}$ is relatively small, in addition to the basic cuts as in eqs. (4.2) and (4.3), we impose the following selection cuts with the better invariant-mass window of $\pm 5 \mathrm{GeV}$

$$
\left|M_{\gamma \gamma}-m_{h}\right|<5 \mathrm{GeV}, \quad p_{T_{\gamma}}>20 \mathrm{GeV}, \quad\left|\eta_{\gamma}\right|<2.5
$$

to substantially reduce the background. The invariant mass distributions for the signal and the continuum backgrounds are shown in figure 9 .

We show the cut flow of cross sections for the signals and backgrounds at the LHC-14 in table 4 . The cross sections shown are calculated with $B$-tagging efficiency $\epsilon_{b}=0.6$, mis-tag probabilities $P_{c \rightarrow b}=0.08$ and $P_{u d s g \rightarrow b}=0.004$, and the jet-fake-photon rate of $10^{-3}$. At the end of the set of cuts, the largest background is the continuum of $t j \gamma \gamma$ followed by $W j j \gamma \gamma$. The largest signal here is obtained with $C_{t}^{S}=-1$ at the order of $35 \times 10^{-3} \mathrm{fb}$, which gives about 10 events with an integrated luminosity of $300 \mathrm{fb}^{-1}$ while the total background has only 2 events, although the signal with $C_{t}^{S}=1$ gives less than 1 event. The significance of the signal given by $S / \sqrt{S+B}$ is also shown in the table. Although in this $\gamma \gamma$ channel the ratio of $S / B$ is better than the $b \bar{b}$ channel, the significance is, however, weaker because of the much fewer signal events.

\subsection{3 $h \rightarrow \tau^{+} \tau^{-}$}

The $\tau^{+} \tau^{-}$channel has been established in the Higgs boson search [26]. The branching ratio for $m_{h}=125-126 \mathrm{GeV}$ is about $6.2-6.3 \times 10^{-2}$ [29]. Since there are always neutrinos in tau-lepton decays, which means that the momentum of the parent tau lepton cannot be fully reconstructed. However, as the tau-lepton momentum is high enough, the visible part 


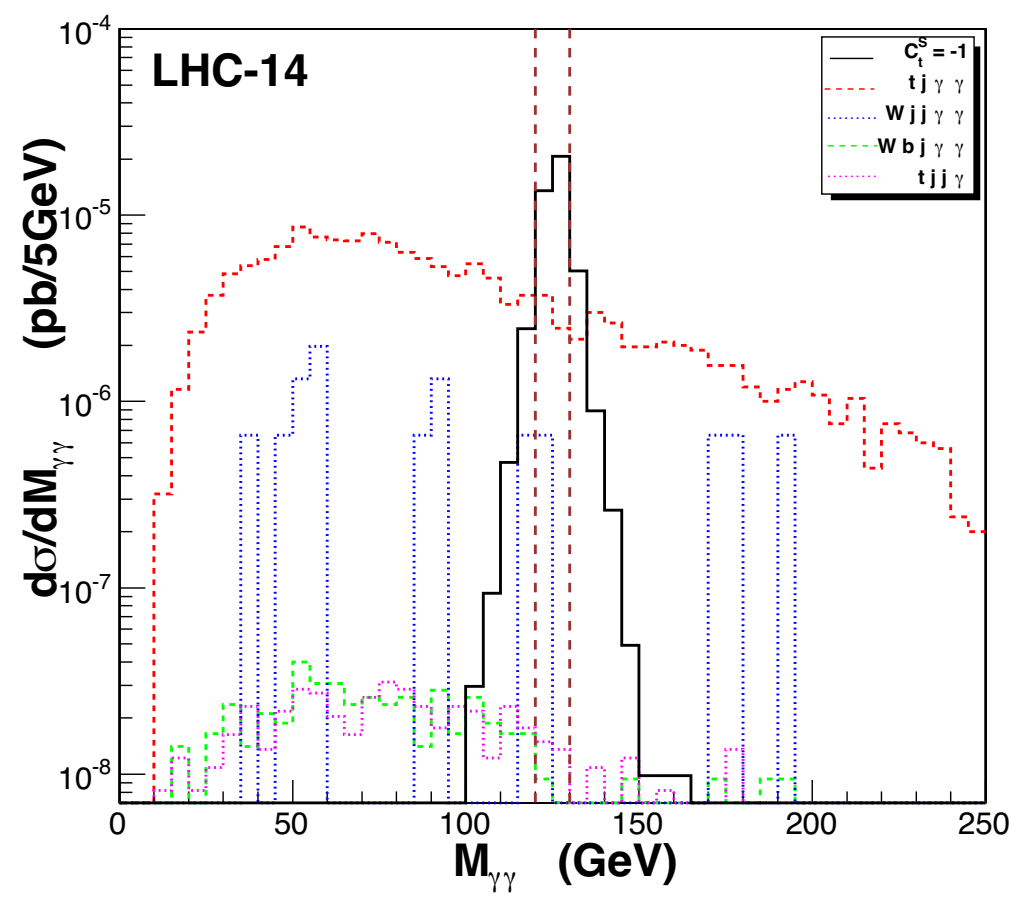

Figure 9. Invariant mass $M_{\gamma \gamma}$ distributions for the signal $p p \rightarrow t h j$ with $C_{t}^{S}=-1$ followed by the semileptonic decay of the top quark and $h \rightarrow \gamma \gamma$, and for various backgrounds listed above at the LHC-14. The shape of the peak for $C_{t}^{S}=1,0$ is the same as the one shown with $C_{t}^{S}=-1$. The selection cuts up to level (3) of table 4 have been applied. The two vertical dashed lines are $m_{h} \pm 5 \mathrm{GeV}$.

of the hadronic tau-lepton decay can be used to determine, to a good approximation, the parent tau-lepton momentum by a rescaling factor (currently the tau-lepton momentum is reconstructed in the jet mode of the tau decay, and the rescaling factor is 1.37 in Delphes 3 [23]). The reconstructed Higgs boson peak using $\tau \tau$ channel is much broader than those using the diphoton and 4-lepton modes: see figure 10. We therefore impose a loose cut in the Higgs-mass window as follows

$$
110 \mathrm{GeV}<M_{\tau \tau}<150 \mathrm{GeV}, \quad p_{T_{\tau}}>25 \mathrm{GeV}, \quad\left|\eta_{\tau}\right|<2.5
$$

The most relevant background is the continuum $t j \tau \tau$ with intermediate $\gamma^{*}$ and $Z$. Another background is $t \bar{t}$ when one of the top decays hadronically and the jets fake the tau-lepton. The $t \bar{t} W$ is also relevant when $W \rightarrow \tau \nu_{\tau}$ and one of the top $t \rightarrow b \tau \nu_{\tau}$. However, these two backgrounds turn out to be very small after cuts.

We show the cut flow of cross sections for the signals and backgrounds at the LHC-14 in table 5 . The cross sections shown are calculated with the $B$-tagging efficiency $\epsilon_{b}=0.6$, $\tau$-tagging efficiency $\epsilon_{\tau}=0.5$, mis-tag probabilities $P_{c \rightarrow b}=0.08$ and $P_{u d s g \rightarrow b}=0.004$, and the jet-fake- $\tau$ rate $P_{j \rightarrow \tau}=0.01$. At the end of the set of cuts, the largest background is the continuum of $t j \tau \tau$. The signal event rates are about 0.5 to 7 with an integrated luminosity 


\begin{tabular}{|lcccccc|}
\hline & \multicolumn{3}{c}{ Signals (fb) } & \multicolumn{5}{c|}{ Backgrounds (fb) } \\
& $C_{t}^{S}=1$ & $C_{t}^{S}=0$ & $C_{t}^{S}=-1$ & $t j \tau \tau$ & $t \bar{t}$ & $t \bar{t} W$ \\
\hline (1) Basic cuts eq. (4.2) & & & & & & \\
and $p_{T_{\tau}}>25 \mathrm{GeV},\left|\eta_{\tau}\right|<2.5$ & 0.00682 & 0.0257 & 0.1026 & 0.0701 & 0.420 & 0.000672 \\
$(2) 2.5<\left|\eta_{j}\right|<4.7$ & 0.00355 & 0.0148 & 0.0585 & 0.0333 & 0.0 & $4.27 \times 10^{-5}$ \\
$(3) M_{b l}<200 \mathrm{GeV}$ & 0.00345 & 0.0141 & 0.0555 & 0.0319 & 0.0 & $4.27 \times 10^{-5}$ \\
$(4) 110<M_{\tau \tau}<150 \mathrm{GeV}$ & 0.00158 & 0.00616 & 0.0244 & 0.0105 & 0.0 & $1.904 \times 10^{-5}$ \\
\hline$S / \sqrt{S+B}$ for $300 \mathrm{fb}^{-1}$ & 0.25 & 0.83 & 2.3 & & & \\
\hline
\end{tabular}

Table 5. The cut flow of cross sections in fb at the LHC-14 for the signal $p p \rightarrow t h j$ with semileptonic decay of the top quark and $h \rightarrow \tau^{+} \tau^{-}$, and various backgrounds. We have used the $B$-tag efficiency $\epsilon_{b}=0.6, \tau$-tagging efficiency $\epsilon_{\tau}=0.5$, mis-tag $P_{c \rightarrow b}=0.08$ and $P_{u d s g \rightarrow b}=0.004$, and the jet-fake rate $P_{j \rightarrow \tau}=0.01$.

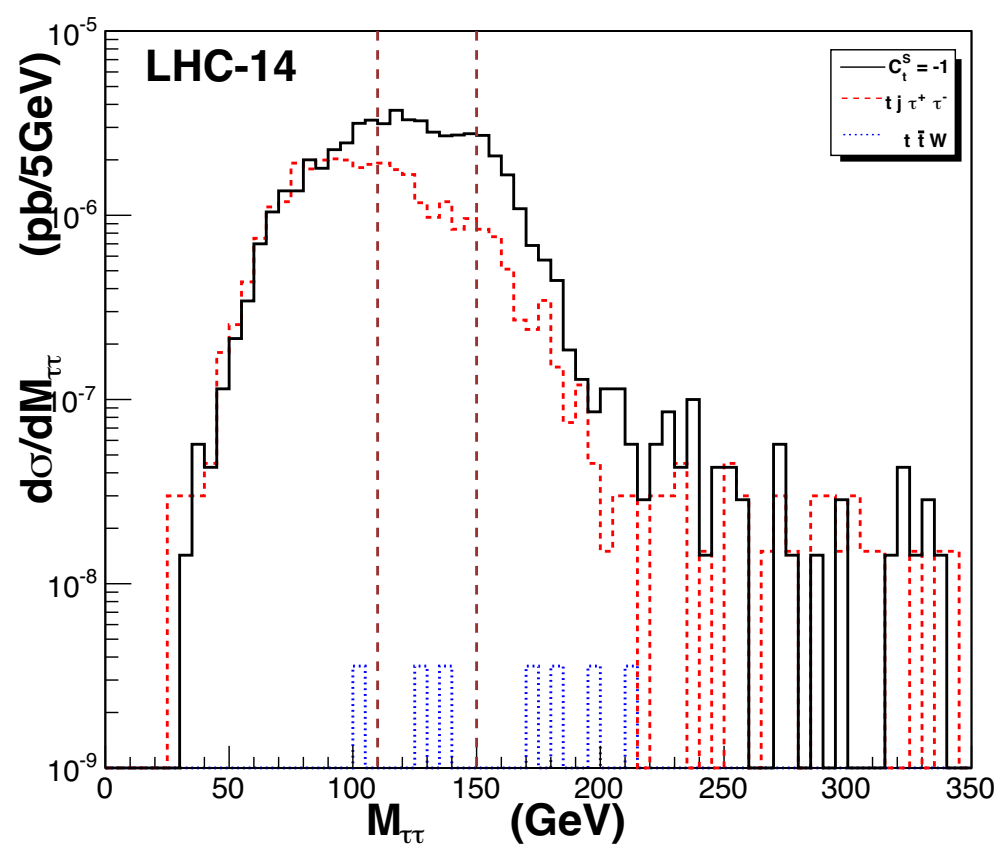

Figure 10. Invariant mass $M_{\tau^{+} \tau^{-}}$distributions for the signal $p p \rightarrow t h j$ with $C_{t}^{S}=-1$ followed by the semileptonic decay of the top quark and $h \rightarrow \tau^{+} \tau^{-}$, and for various backgrounds listed above at the LHC-14. The shape of the peak for $C_{t}^{S}=1,0$ is the same as the one shown with $C_{t}^{S}=-1$. The selection cuts up to level (3) of table 5 have been applied. The two vertical dashed lines are at 110 and $150 \mathrm{GeV}$.

of $300 \mathrm{fb}^{-1}$ for $C_{t}^{S}=1$ to -1 , and also the significance $S / \sqrt{S+B}$ ranges from 0.25 to 2.3 for $C_{t}^{S}=1$ to -1 . The significance level is inferior to both the $b \bar{b}$ and $\gamma \gamma$ modes, mainly because of the smaller branching ratio and the lower $\tau$ identification efficiency. 


\subsection{Hadronic top decay}

In this subsection, we consider associated Higgs production with a single top quark and a forward jet, followed by the hadronic decay of the top quark:

$$
p p \longrightarrow t h j \longrightarrow\left(b j_{1} j_{2}\right) h j,
$$

where we label $j_{1}, j_{2}$ for the 2 jets from the top-quark decay. We first apply the same basic cuts as in eq. (4.2) on identifying the $b$ quark and the 2 jets from the top decay, as well as the forward jet, which has the further requirement

$$
2.5<\left|\eta_{j}\right|<4.7
$$

We also impose the requirement on the $b$ quark and the two jets that originate from the top decay by

$$
M_{b j_{1} j_{2}}<300 \mathrm{GeV} \text {. }
$$

\subsection{1 $h \rightarrow Z Z^{*} \rightarrow 4 \ell$}

The Higgs boson decaying into $Z Z^{*} \rightarrow 4 \ell$ is one of the cleanest channels for discovery and reconstruction. Since the branching ratio into $4 \ell$ is very small and the $p_{T}$ of the electrons or muons is only of order $20 \mathrm{GeV}$, and so we apply mild cuts for the charged leptons

$$
p_{T_{\ell}}>5 \mathrm{GeV}, \quad\left|\eta_{\ell}\right|<2.5 .
$$

We further apply the Higgs-mass window cut on the invariant mass formed by the 4 charged leptons

$$
\left|M_{4 \ell}-m_{h}\right|<5 \mathrm{GeV} .
$$

The invariant mass distributions for the signal and various backgrounds are shown in figure 11.

We show the cut flow of cross sections for the signals and backgrounds at the LHC-14 in table 6 . The cross sections shown are calculated with $B$-tagging efficiency $\epsilon_{b}=0.6$, mis-tag probabilities $P_{c \rightarrow b}=0.08$ and $P_{u d s g \rightarrow b}=0.004$. At the end of the set of cuts, the largest background comes from $t j 4 \ell$, where the $\ell \ell \ell \ell$ comes from the $\gamma^{*}$ and $Z^{*}$ exchanges, but it is rendered extremely small. However, the signal event rates are also very tiny, substantially smaller than 1 event for an integrated luminosity of $300 \mathrm{fb}^{-1}$. Nevertheless, if integrated luminosity can increase to $3000 \mathrm{fb}^{-1}$ we can have $2-3$ events for $C_{t}^{S}=-1$. The event rate is small simply because of the tiny branching ratio of $h \rightarrow Z Z^{*} \rightarrow 4 \ell$. One perhaps can perform the calculation using the $Z Z^{*} \rightarrow \ell^{+} \ell^{-} j j$ mode, but we shall delay this channel in future works.

\subsection{Distinction among $C_{t}^{S}=1,0,-1$}

In addition to the differences in cross section, we further found that the spatial separations $\Delta R$ among the forward jet, the $b$ quark, the charged lepton, and the reconstructed Higgs boson show interesting differences among $C_{t}^{S}=1,0,-1$, as shown in figure 12 . Without loss of generality we use the $h \rightarrow \gamma \gamma$ decay mode for this study, because the 4-momentum 


\begin{tabular}{|lcccccc|}
\hline & \multicolumn{3}{c}{ Signals $\left(10^{-3} \mathrm{fb}\right)$} & \multicolumn{3}{c|}{ Backgrounds $\left(10^{-3} \mathrm{fb}\right)$} \\
& $C_{t}^{S}=1$ & $C_{t}^{S}=0$ & $C_{t}^{S}=-1$ & $t j 4 \ell$ & $Z Z 3 j$ & $Z Z b 2 j$ \\
\hline (1) Basic cuts eq. (4.2) & & & & & & \\
and $p_{T_{j_{1,2}}}>25 \mathrm{GeV},\left|\eta_{j_{1,2}}\right|<2.5$ & & & & & & \\
but with $p_{T_{\ell}}>5 \mathrm{GeV}$ & 0.136 & 0.531 & 1.77 & 0.955 & 20.1 & 10.0 \\
$(2) 2.5<\left|\eta_{j}\right|<4.7$ & 0.091 & 0.366 & 1.18 & 0.539 & 8.01 & 5.01 \\
$(3) M_{b j_{1} j_{2}}<300 \mathrm{GeV}$ & 0.081 & 0.324 & 1.02 & 0.438 & 3.79 & 1.97 \\
$(4)\left|M_{4 \ell}-m_{h}\right|<5 \mathrm{GeV}$ & 0.072 & 0.289 & 0.901 & $8.65 \times 10^{-3}$ & 0.0 & 0.0 \\
\hline$S / \sqrt{S+B}$ for $300 \mathrm{fb}^{-1}$ & 0.14 & 0.29 & 0.52 & & & \\
\hline
\end{tabular}

Table 6. The cut flow of cross sections in $10^{-3} \mathrm{fb}$ at the LHC-14 for the signal $p p \rightarrow$ th $j$ with hadronic decay of the top quark and $h \rightarrow Z Z^{*} \rightarrow 4 \ell$, and various backgrounds. We have used a $B$-tag efficiency $\epsilon_{b}=0.6$, mis-tag $P_{c \rightarrow b}=0.08$ and $P_{u d s g \rightarrow b}=0.004$.

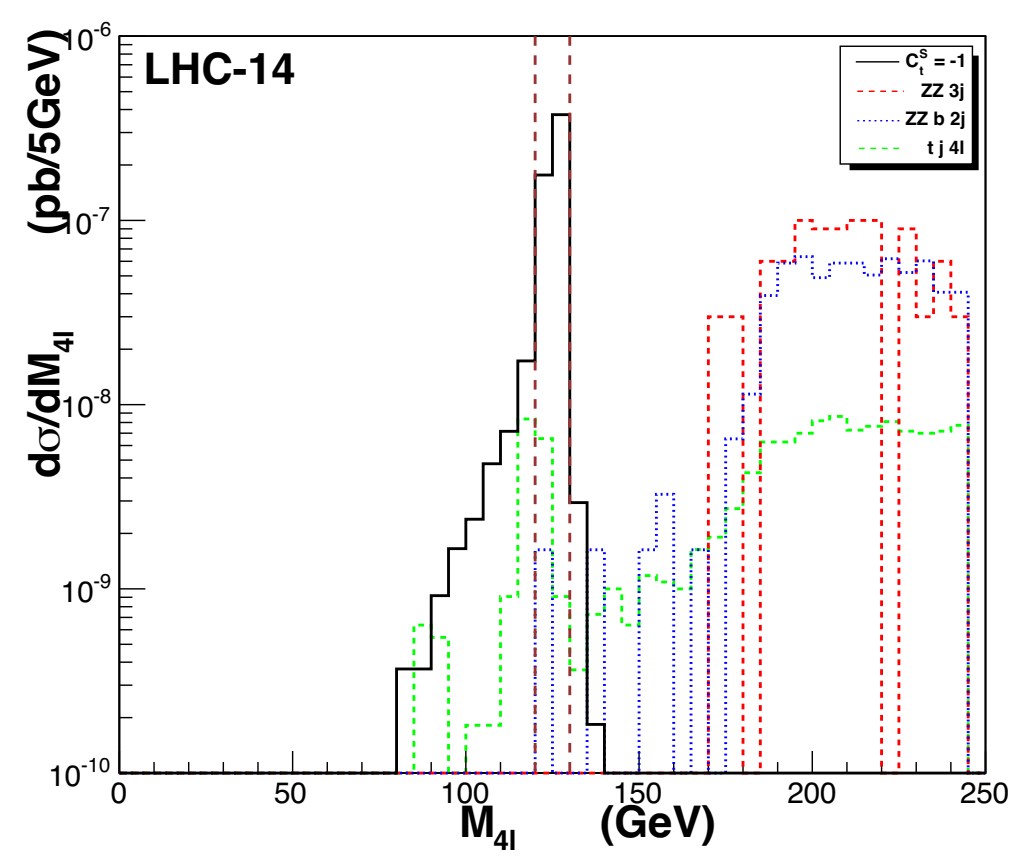

Figure 11. Invariant mass $M_{4 \ell}$ distributions for the signal $p p \rightarrow t h j$ with $C_{t}^{S}=-1$ followed by the hadronic decay of the top quark and $h \rightarrow Z Z^{*} \rightarrow 4 \ell$, and for various backgrounds listed above at the LHC-14. The shape of the peak for $C_{t}^{S}=1,0$ is the same as the one shown with $C_{t}^{S}=-1$. The selection cuts up to level (3) of table 6 have been applied. The two vertical dashed lines are $m_{h} \pm 5 \mathrm{GeV}$.

of the Higgs boson can be reconstructed cleanly by summing the 4-momenta of the two photons. Since the behavior of the charged lepton and the $b$ quark coming from the top quark decay is similar, we choose only the charged lepton to show the $\Delta R$ distributions. We found that the spatial separation $\Delta R$ between the forward jet and the Higgs boson becomes wider when $C_{t}^{S}$ deviates from the SM value 1, while that between the charged lepton and 

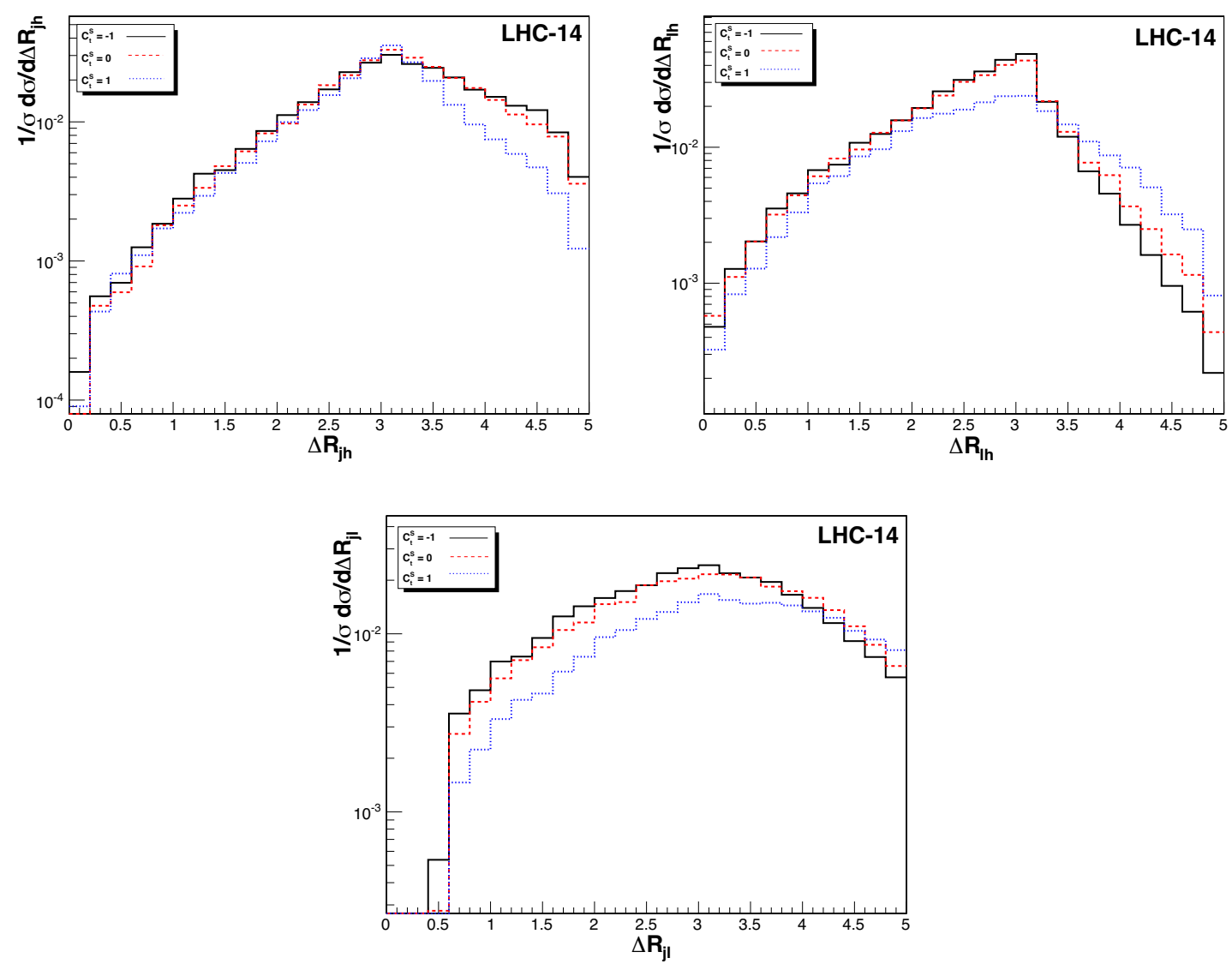

Figure 12. Normalized $\Delta R$ distributions for various pairs of particles $(\ell, j, h)$, where the momentum of $h$ is reconstructed by the photon pair, for the signal process $p p \rightarrow$ th $j$ with $C_{t}^{S}=-1,0,1$ followed by the semileptonic decay of the top quark and $h \rightarrow \gamma \gamma$ at the LHC-14. Behavior of $b$ and $\ell$ is about the same, as they are coming from the same top quark decay. We need only one of them: $\ell$.

the Higgs boson and that between the forward jet and the charged lepton become narrower as $C_{t}^{S}$ deviates from 1. Similar patterns were observed in ref. [14]. It was also shown in ref. [13] that the variations in scalar and pseudoscalar components of the top-Yukawa coupling can also induce interesting angular correlations.

\section{Discussion}

We have studied associated Higgs production with a single top quark in the dominant process $p p \rightarrow t h j$, followed by the semileptonic decay of the top quark and $h \rightarrow b \bar{b}, \gamma \gamma, \tau^{+} \tau^{-}$ and by the hadronic decay of the top quark and $h \rightarrow Z Z^{*} \rightarrow 4 \ell$. So far, we have found that the $h \rightarrow b \bar{b}$ channel offers the best chance in terms of significance $S / \sqrt{S+B}$ for observing the signal with various $C_{t}^{S}$. When $C_{t}^{S}=1(\mathrm{SM})$ the significance level is very low at 0.4 , but it quickly rises to a large enough value 5.6 when $C_{t}^{S}=-1$. The signal event rates are from 18 to 270 with an integrated luminosity of $300 \mathrm{fb}^{-1}$. In figure 13 , we show the 


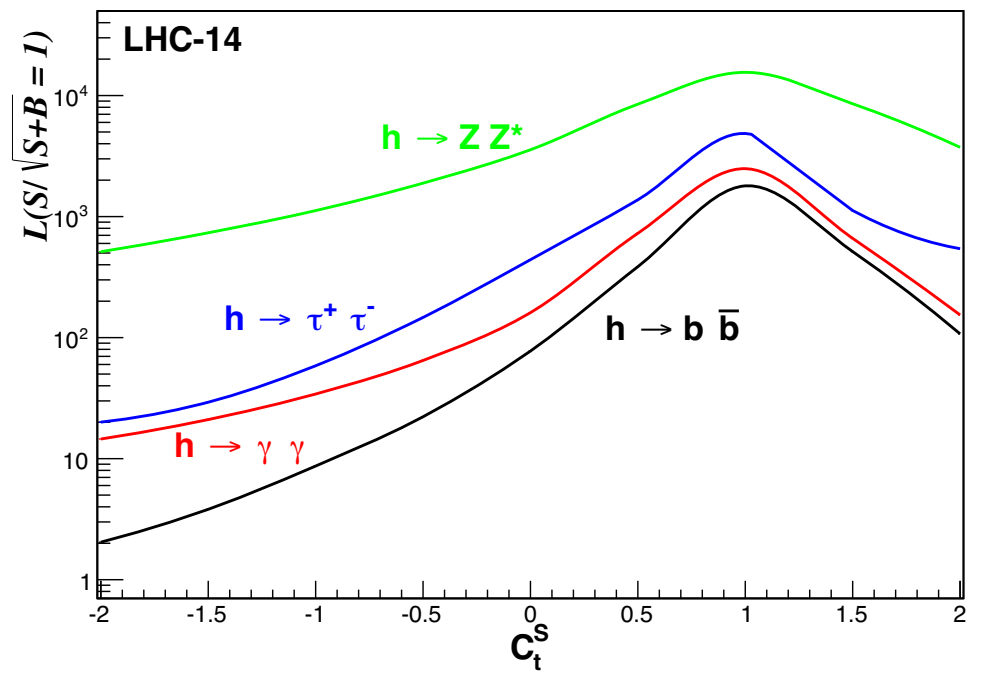

Figure 13. Required luminosities at the LHC-14 for the process $p p \rightarrow t h j$ in various decay channels of the Higgs boson to achieve $S / \sqrt{S+B}=1$. We show the channels $h \rightarrow b \bar{b}, \gamma \gamma, \tau^{+} \tau^{-}$, and $Z Z^{*} \rightarrow 4 \ell$.

required luminosities to achieve a significance level of $S / \sqrt{S+B}=1$ for various channels considered in this work versus $C_{t}^{S}=-2$ to 2 . Note that $S / \sqrt{S+B}>1$ implies

$$
S>1+\frac{B}{S}>1
$$

i.e., the event rate $S>1$ is guaranteed. The best channel is the $h \rightarrow b \bar{b}$. The second and the third are $h \rightarrow \gamma \gamma$ and $h \rightarrow \tau^{+} \tau^{-}$, respectively. The last one is the $h \rightarrow Z Z^{*} \rightarrow 4 \ell$. Note that the $Z Z^{*}$ channel requires a very large luminosity in order to achieve $S / \sqrt{S+B}>1$ simply because of its very small signal cross sections. Similarly, because of the small signal cross sections in $\gamma \gamma$ channel, the $h \rightarrow \gamma \gamma$ channel requires larger luminosities than the $b \bar{b}$ channel in order to achieve $S / \sqrt{S+B}>1$, although the $S / B$ ratio is much better in the $\gamma \gamma$ than in the $b \bar{b}$ channel.

Before we close we offer the following comments.

1. In the current framework, the bottom-Yukawa has very small effects on the cross section, because the bottom-Yukawa coupling $C_{b}^{S}$ is approximately constrained to be within the range -2 to $+2[3]$.

2. The higher order process, $p p \rightarrow t h b j$, contains one more $b$ quark in the final state. Potentially, it can increase the signal sensitivity based on a parton-level study [11]. However, we have shown in this work that with full detector simulations the Higgsmass window cut is not as effective as that in parton-level. Furthermore, there are further combinatorics problems as we have to identify the $b$ quark from top decay and the two $b$ quarks from the Higgs boson decay. 
3. The $h \rightarrow b \bar{b}$ decay mode turns out to be the best in terms of significance to probe associated Higgs production with a single top quark, because of the larger event rates, although the signal-to-background ratio is less than 1.

4. The best signal-to-background ratio $S / B$ is achieved in the $h \rightarrow Z Z^{*} \rightarrow 4 \ell$ channel, followed by the $\gamma \gamma, \tau^{+} \tau^{-}$, and $b \bar{b}$ channels. Nevertheless, the event rates in $4 \ell$ and $\gamma \gamma$ are too low for detection.

5. Better $\tau$-lepton identification is needed in order to raise the efficiency in identifying the $h \rightarrow \tau \tau$ decay. Perhaps, one can look into the substructure in the fast-moving $\tau$ jet. If one can achieve better efficiencies, it will enhance the probe of the single top associated Higgs production.

6. The $h \rightarrow Z Z^{*} \rightarrow 4 \ell$ has a very small branching ratio, and so the detection of single top associated Higgs production requires an extremely high luminosity. One should pursue the $Z Z^{*} \rightarrow \ell^{+} \ell^{-} j j$ mode, which has about one order of magnitude larger in event rates, but different backgrounds.

7. We do not attempt the $h \rightarrow W W^{*}$ mode in this work, simply because the Higgs boson peak cannot be reconstructed in this mode, unless we go for the $4 j$ mode.

8. If the top-Yukawa is close to the SM value, the best chance to observe associated Higgs production with a single top quark is via the $h \rightarrow b \bar{b}$ channel. However, it requires an integrated luminosity more than $1000 \mathrm{fb}^{-1}$.

In summary, we have studied the effects of varying the top-Yukawa coupling in the Higgs associated production with a single top quark, with full detector simulations, We found that the $h \rightarrow b \bar{b}$ mode with the semileptonic decay of the top quark has the highest potential in observing the process and the effects of top-Yukawa coupling, especially the sign of the top-Yukawa coupling can be determined.

\section{Acknowledgments}

This work was supported by the National Science Council of Taiwan under Grants No. 1022112-M-007-015-MY3, and by the National Research Foundation of Korea (NRF) grant (No. 2013R1A2A2A01015406). This study was also financially supported by Chonnam National University, 2012. J.S.L thanks National Center for Theoretical Sciences (Hsinchu, Taiwan) for the great hospitality extended to him while this work was being performed.

\section{A Amplitude of $q b \rightarrow t h q^{\prime}$ in the effective $W$ approximation}

In this appendix, we present the amplitude of the process $q b \rightarrow t h q^{\prime}$ in the effective $W$ approximation assuming $h$ is a spin-zero CP-mixed state. In this process, the dominant contribution comes from the region where the $W$ boson emitted from the incoming quark 
$q$ is close to onshell and one can approximately represent the process by the $W$ boson scattering with the incoming $b$ quark to give $h$ and $t$ in the final state:

$$
W\left(p_{W}\right) b\left(p_{b}\right) \rightarrow h\left(p_{h}\right) t\left(p_{t}\right) .
$$

The process $W b \rightarrow h t$ receives contributions from $(a)$ a $t$-channel diagram with the $W$ exchange and (b) a $s$-channel diagram with the $t$ exchange. The vertex factor for $h W W$ is given in eq. (2.1) and that for $h t \bar{t}$ in eq. (2.2) with the identification of $g_{h W W}=C_{v}$ and $g_{h t t}^{S, P}=C_{t}^{S, P}$, see eq. (2.7). Then, the amplitude of each diagram reads

$$
\begin{aligned}
& \mathcal{M}_{(a)}=\frac{g^{2} m_{t} C_{v}}{\sqrt{2} m_{W}\left(t-m_{W}^{2}\right)}\left[\left(p_{b}-p_{t}\right) \cdot \epsilon\left(p_{W}\right) \bar{u}\left(p_{t}\right) P_{L} u\left(p_{b}\right)+\frac{m_{W}^{2}}{m_{t}} \bar{u}\left(p_{t}\right) \notin\left(p_{W}\right) P_{L} u\left(p_{b}\right)\right], \quad(\mathrm{A} .2) \\
& \mathcal{M}_{(b)}=\frac{g^{2} m_{t}}{\sqrt{2} m_{W}\left(s-m_{t}^{2}\right)}\left[m_{t} C_{t}^{S} \bar{u}\left(p_{t}\right) \notin\left(p_{W}\right) P_{L} u\left(p_{b}\right)+\left(\frac{C_{t}^{S}-i C_{t}^{P}}{2}\right) \bar{u}\left(p_{t}\right) p_{h} \phi\left(p_{W}\right) P_{L} u\left(p_{b}\right)\right],
\end{aligned}
$$

where $s=\left(p_{b}+p_{W}\right)^{2}=\left(p_{t}+p_{h}\right)^{2}, t=\left(p_{b}-p_{t}\right)^{2}=\left(p_{h}-p_{W}\right)^{2}$, and $u=\left(p_{b}-p_{h}\right)^{2}=$ $\left(p_{t}-p_{W}\right)^{2}$ and $\epsilon^{\mu}\left(p_{W}\right)$ denotes the polarization vector of $W$ boson. In the high-energy limit of $s,|t|,|u| \gg m_{W}^{2}, m_{h}^{2}, m_{t}^{2}$, we find that

$$
\mathcal{M}=\mathcal{M}_{(a)}+\mathcal{M}_{(b)} \approx-\frac{g^{2} m_{t}}{2 \sqrt{2} m_{W}^{2}}\left[\left(C_{v}-C_{t}^{S}\right)+i C_{t}^{P}\right] \bar{u}\left(p_{t}\right) P_{L} u\left(p_{b}\right)
$$

taking the longitudinally polarized $W$ or $\epsilon^{\mu}\left(p_{W}\right) \approx p_{W}^{\mu} / m_{W}$ and using $\left(p_{b}-p_{t}\right) \cdot \epsilon\left(p_{W}\right) \approx$ $-t /\left(2 m_{W}\right)$ and $\bar{u}\left(p_{t}\right) \not p_{h} \notin\left(p_{W}\right) P_{L} u\left(p_{b}\right) \approx\left(s / m_{W}\right) \bar{u}\left(p_{t}\right) P_{L} u\left(p_{b}\right)$. We observe our results are consistent with those given in ref. [30]. We note that, in the high-energy limit,

$$
\overline{|\mathcal{M}|^{2}} \propto\left[\left(C_{v}-C_{t}^{S}\right)^{2}+\left(C_{t}^{P}\right)^{2}\right](-t)
$$

and therefore the absence of this unitarity-breaking term requires $C_{v}=C_{t}^{S}$ and $C_{t}^{P}=0$.

Open Access. This article is distributed under the terms of the Creative Commons Attribution License (CC-BY 4.0), which permits any use, distribution and reproduction in any medium, provided the original author(s) and source are credited.

\section{References}

[1] ATLAS collaboration, Observation of a new particle in the search for the Standard Model Higgs boson with the ATLAS detector at the LHC, Phys. Lett. B 716 (2012) 1 [arXiv: 1207.7214] [INSPIRE].

[2] CMS collaboration, Observation of a new boson at a mass of $125 \mathrm{GeV}$ with the CMS experiment at the LHC, Phys. Lett. B 716 (2012) 30 [arXiv:1207.7235] [InSPIRE].

[3] K. Cheung, J.S. Lee and P.-Y. Tseng, Higgs Precision (Higgcision) Era begins, JHEP 05 (2013) 134 [arXiv:1302.3794] [INSPIRE].

[4] P.W. Higgs, Broken Symmetries and the Masses of Gauge Bosons, Phys. Rev. Lett. 13 (1964) 508 [INSPIRE]. 
[5] F. Englert and R. Brout, Broken Symmetry and the Mass of Gauge Vector Mesons, Phys. Rev. Lett. 13 (1964) 321 [INSPIRE].

[6] G.S. Guralnik, C.R. Hagen and T.W.B. Kibble, Global Conservation Laws and Massless Particles, Phys. Rev. Lett. 13 (1964) 585 [InSPIRE].

[7] T.M.P. Tait and C.-P. Yuan, Single top quark production as a window to physics beyond the standard model, Phys. Rev. D 63 (2000) 014018 [hep-ph/0007298] [INSPIRE].

[8] V. Barger, M. McCaskey and G. Shaughnessy, Single top and Higgs associated production at the LHC, Phys. Rev. D 81 (2010) 034020 [arXiv:0911.1556] [INSPIRE].

[9] S. Biswas, E. Gabrielli and B. Mele, Single top and Higgs associated production as a probe of the Htt coupling sign at the LHC, JHEP 01 (2013) 088 [arXiv:1211.0499] [INSPIRE].

[10] S. Biswas, E. Gabrielli, F. Margaroli and B. Mele, Direct constraints on the top-Higgs coupling from the $8 \mathrm{TeV}$ LHC data, JHEP 07 (2013) 073 [arXiv:1304.1822] [INSPIRE].

[11] M. Farina, C. Grojean, F. Maltoni, E. Salvioni and A. Thamm, Lifting degeneracies in Higgs couplings using single top production in association with a Higgs boson, JHEP 05 (2013) 022 [arXiv:1211.3736] [INSPIRE].

[12] P. Agrawal, S. Mitra and A. Shivaji, Effect of Anomalous Couplings on the Associated Production of a Single Top Quark and a Higgs Boson at the LHC, JHEP 12 (2013) 077 [arXiv: 1211.4362] [INSPIRE].

[13] J. Ellis, D.S. Hwang, K. Sakurai and M. Takeuchi, Disentangling Higgs-Top Couplings in Associated Production, JHEP 04 (2014) 004 [arXiv:1312.5736] [INSPIRE].

[14] C. Englert and E. Re, Bounding the top Yukawa with Higgs-associated single-top production, Phys. Rev. D 89 (2014) 073020 [arXiv: 1402.0445] [InSPIRE].

[15] J.S. Lee et al., CPsuperH: A Computational tool for Higgs phenomenology in the minimal supersymmetric standard model with explicit CP-violation, Comput. Phys. Commun. 156 (2004) 283 [hep-ph/0307377] [INSPIRE].

[16] J. Alwall, M. Herquet, F. Maltoni, O. Mattelaer and T. Stelzer, MadGraph 5: Going Beyond, JHEP 06 (2011) 128 [arXiv:1106.0522] [INSPIRE].

[17] P.M. Nadolsky et al., Implications of CTEQ global analysis for collider observables, Phys. Rev. D 78 (2008) 013004 [arXiv:0802.0007] [INSPIRE].

[18] B.W. Harris, E. Laenen, L. Phaf, Z. Sullivan and S. Weinzierl, The Fully differential single top quark cross-section in next to leading order QCD, Phys. Rev. D 66 (2002) 054024 [hep-ph/0207055] [INSPIRE].

[19] N. Kidonakis, Higher-order soft gluon corrections in single top quark production at the LHC, Phys. Rev. D 75 (2007) 071501 [hep-ph/0701080] [INSPIRE].

[20] J.M. Campbell, R. Frederix, F. Maltoni and F. Tramontano, Next-to-Leading-Order Predictions for t-Channel Single-Top Production at Hadron Colliders, Phys. Rev. Lett. 102 (2009) 182003 [arXiv:0903.0005] [INSPIRE].

[21] S. Heim, Q.-H. Cao, R. Schwienhorst and C.-P. Yuan, Next-to-leading order QCD corrections to s-channel single top quark production and decay at the LHC, Phys. Rev. D 81 (2010) 034005 [arXiv: 0911.0620] [INSPIRE]. 
[22] J. Alwall and the CP3 development team, The MG/ME Pythia-PGS package; the Madgraph at http://madgraph.hep.uiuc.edu/; Pythia at https://pythia6.hepforge.org/; and PGS at http://www.physics.ucdavis.edu/ conway/research/software/pgs/pgs4-general.htm.

[23] DELPHES 3 collaboration, J. de Favereau et al., DELPHES 3, A modular framework for fast simulation of a generic collider experiment, JHEP 02 (2014) 057 [arXiv:1307.6346] [INSPIRE].

[24] CMS collaboration, Identification of b-quark jets with the CMS experiment, 2013 JINST 8 P04013 [arXiv: 1211.4462] [INSPIRE].

[25] CMS collaboration, Performance of tau-lepton reconstruction and identification in CMS, 2012 JINST 7 P01001 [arXiv:1109.6034] [INSPIRE].

[26] CMS collaboration, Evidence for the $125 \mathrm{GeV}$ Higgs boson decaying to a pair of $\tau$ leptons, arXiv: 1401.5041 [INSPIRE].

[27] ATLAS collaboration, Expected photon performance in the ATLAS experiment, ATL-PHYS-PUB-2011-007 (2011).

[28] V.D. Barger, K.-m. Cheung, T. Han, J. Ohnemus and D. Zeppenfeld, A Comparative study of the benefits of forward jet tagging in heavy Higgs production at the SSC, Phys. Rev. D 44 (1991) 1426 [INSPIRE].

[29] LHC Higgs Cross section Working Group, https://twiki.cern.ch/twiki/bin/view/LHCPhysics/Cross sections.

[30] F. Maltoni, K. Paul, T. Stelzer and S. Willenbrock, Associated production of Higgs and single top at hadron colliders, Phys. Rev. D 64 (2001) 094023 [hep-ph/0106293] [INSPIRE]. 